\title{
Graphs with large distinguishing chromatic number
}

\author{
Michael Cavers* \\ Department of Mathematics and Statistics \\ University of Calgary \\ Calgary, Alberta, Canada, T2N 1N4 \\ michael. cavers@ucalgary.ca
}

\author{
Karen Seyffarth \\ Department of Mathematics and Statistics \\ University of Calgary \\ Calgary, Alberta, Canada, T2N 1N4 \\ kseyffar@math.ucalgary.ca
}

Submitted: Jun 3, 2012; Accepted: Jan 16, 2013; Published: Jan 29, 2013

Mathematics Subject Classifications: 05C15, 05C25.

\begin{abstract}
The distinguishing chromatic number $\chi_{D}(G)$ of a graph $G$ is the minimum number of colours required to properly colour the vertices of $G$ so that the only automorphism of $G$ that preserves colours is the identity. For a graph $G$ of order $n$, it is clear that $1 \leqslant \chi_{D}(G) \leqslant n$, and it has been shown that $\chi_{D}(G)=n$ if and only if $G$ is a complete multipartite graph. This paper characterizes the graphs $G$ of order $n$ satisfying $\chi_{D}(G)=n-1$ or $\chi_{D}(G)=n-2$.
\end{abstract}

\section{Introduction}

Unless otherwise specified, this paper uses the notation and terminology of [2]. A labelling of a graph $G$ is an assignment of labels to the vertices of $G$. A colouring of $G$ is a special case of a labelling where no two adjacent vertices share the same label (in this context we refer to the labels as colours). Note that some authors call such a labelling a proper colouring whereas in this paper we simply use the term colouring. A labelling of $G$ is said to be distinguishing provided that no automorphism of $G$ (other than the identity) preserves the labels. The distinguishing number of $G$, denoted by $D(G)$, is the minimum number of labels required to produce a labelling of $G$ that is distinguishing, while the chromatic number of $G$, denoted by $\chi(G)$, is the minimum number of colours required to produce a colouring of $G$. The distinguishing chromatic number of $G$, denoted by $\chi_{D}(G)$, is the minimum number of colours required to produce a distinguishing colouring of $G$.

The neighbourhood of a vertex $v \in V(G)$, denoted by $N_{G}(v)$, is the set of all vertices of $G$ that are adjacent to $v$. If $x$ and $y$ are vertices of $G$, we use the notation $x \sim y$ if

\footnotetext{
*Supported by an NSERC PDF.
} 
$x y$ is an edge of $G$, and the notation $x \neq y$ if $x y$ is not an edge of $G$. For disjoint sets $A, B \subseteq V(G)$, we write $A \sim B$ (respectively $A \nsim B$ ) to mean that for every $a \in A$ and every $b \in B, a \sim b$ (respectively $a \not b$ ) holds. In the case that $A=\{a\}$ we simply write $a \sim B$ (respectively $a \nsim B$ ).

A coloured graph is an ordered pair $(G, f)$ where $G$ is a graph and $f$ is a colouring function mapping vertices of $G$ to a set of colours. A colour class $\mathcal{C}_{r}$ of $(G, f)$ is a set of all vertices of $(G, f)$ with the same colour $r$. A singleton (respectively doubleton) colour class consists of precisely one vertex (respectively two vertices). If $f$ is a colouring of $G$, then an automorphism $\sigma$ of $G$ is called colour preserving if $f(\sigma(v))=f(v)$, for every $v \in V(G)$. A vertex $v \in V(G)$ is pinned (under the colouring $f$ ) if $\sigma(v)=v$, for every colour preserving automorphism $\sigma$ of $G$.

A subgraph $H$ of a graph $G$ is said to be induced if for any pair of vertices $x$ and $y$ of $H, x y$ is an edge of $H$ if and only if $x y$ is an edge of $G$. If $S \subseteq V(G)$ is the vertex set of $H$, then $H$ can be written as $G[S]$ and is said to be induced by $S$. We use the notation $G \backslash H$ to denote the induced subgraph $G[V(G) \backslash V(H)]$.

The distinguishing chromatic number has been analyzed (and in some cases computed) for various classes of graphs, such as paths, cycles, generalized Petersen graphs, hypercubes, interval graphs, planar graphs, trees and bipartite graphs (see $[6,9,10,11,12,13$, 14]). In particular, let $P_{n}$ denote a path with $n$ vertices and $C_{n}$ a cycle with $n$ vertices. It is shown in [6] that

$\chi_{D}\left(P_{n}\right)=\left\{\begin{array}{ll}2, & \text { for } n \geqslant 2 \text { and } n \text { even, } \\ 3, & \text { for } n \geqslant 3 \text { and } n \text { odd, }\end{array}\right.$ and $\chi_{D}\left(C_{n}\right)= \begin{cases}3, & \text { for } n=3,5 \text { or } n \geqslant 7, \\ 4, & \text { for } n=4 \text { or } n=6 .\end{cases}$

The distinguishing chromatic number has also been discussed in the context of Cartesian products, graph joins and other binary operations on graphs (see $[4,5,6,8]$ ).

An obvious bound on the distinguishing chromatic number of a graph $G$ of order $n$ is $1 \leqslant \chi_{D}(G) \leqslant n$. In [6], the graphs that satisfy the upper bound with equality are characterized.

Theorem 1.1 [6, Theorem 2.3] Let $G$ be a graph of order $n$. Then $\chi_{D}(G)=n$ if and only if $G$ is a complete multipartite graph.

Clearly, any graph $G$ of order $n \geqslant 2$ must satisfy $\chi_{D}(G) \geqslant 2$. Characterizing graphs with $\chi_{D}(G)=2$ is an interesting but hard open problem (see [7]). In this paper, we investigate graphs with large distinguishing chromatic number and in Section 3, we characterize the graphs $G$ of order $n$ that satisfy $\chi_{D}(G)=n-1$ or $\chi_{D}(G)=n-2$. In order to complete this characterization we present some properties of graphs with large distinguishing chromatic number in Section 2. In particular, lower bounds on the distinguishing chromatic number of induced subgraphs of a graph are given and applied to obtain a list of forbidden induced subgraphs of graphs $G$ with $\chi_{D}(G) \geqslant|V(G)|-2$. 


\section{Preliminary results}

We first provide an upper bound on $\chi_{D}(G)$ in terms of the distinguishing chromatic numbers of a collection of disjoint induced subgraphs of $G$ that span $V(G)$.

Lemma 2.1 Let $G$ be a graph. If $V(G)=S_{1} \cup S_{2} \cup \cdots \cup S_{k}$ such that $S_{i} \cap S_{j}=\emptyset$, for all $i \neq j$, then

$$
\chi_{D}(G) \leqslant \sum_{i=1}^{k} \chi_{D}\left(G\left[S_{i}\right]\right) .
$$

In particular, if $H$ is an induced subgraph of $G$, then

$$
\chi_{D}(G) \leqslant \chi_{D}(H)+\chi_{D}(G \backslash H) .
$$

Proof. For each $i$, let $f_{i}: S_{i} \rightarrow\left\{1,2, \ldots, \chi_{D}\left(G\left[S_{i}\right]\right)\right\}$ be a distinguishing colouring of $G\left[S_{i}\right]$ using $\chi_{D}\left(G\left[S_{i}\right]\right)$ labels. Then the labelling that assigns each vertex $v \in S_{i}$ the label $\left(i, f_{i}(v)\right)$ is a distinguishing colouring of $G$ using $\sum_{i=1}^{k} \chi_{D}\left(G\left[S_{i}\right]\right)$ colours.

One occurrence of equality in Lemma 2.1 is for the join of graphs. In particular, the join of pairwise disjoint graphs $G_{1}, G_{2}, \ldots, G_{k}$, denoted by $\bigvee_{i=1}^{k} G_{i}$, is the graph with vertex set $V\left(G_{1}\right) \cup V\left(G_{2}\right) \cup \cdots \cup V\left(G_{k}\right)$ and edge set $E\left(G_{1}\right) \cup E\left(G_{2}\right) \cup \cdots \cup E\left(G_{k}\right) \cup\{x y$ : $\left.x \in V\left(G_{i}\right), y \in V\left(G_{j}\right), i \neq j\right\}$.

Remark 2.2 [6, Observation 2.1(3)] Let $G_{1}, G_{2}, \ldots, G_{k}$ be pairwise disjoint graphs and let $G=\bigvee_{i=1}^{k} G_{i}$. Then

$$
\chi_{D}(G)=\sum_{i=1}^{k} \chi_{D}\left(G_{i}\right) .
$$

An instance where Lemma 2.1 may provide a weak bound is the (disjoint) union of graphs. In particular, the union of pairwise disjoint graphs $G_{1}, G_{2}, \ldots, G_{k}$, denoted by $\bigcup_{i=1}^{k} G_{i}$, is the graph with vertex set $V\left(G_{1}\right) \cup V\left(G_{2}\right) \cup \cdots \cup V\left(G_{k}\right)$ and edge set $E\left(G_{1}\right) \cup E\left(G_{2}\right) \cup \cdots \cup E\left(G_{k}\right)$. For a graph $H$, we denote $\bigcup_{i=1}^{k} H$ simply as $k H$.

As in [3], if $f$ and $g$ are two colourings of a graph $G$, then $f$ and $g$ are said to be equivalent if there is an automorphism of $G$ that maps $(G, f)$ to $(G, g)$. Let $\mathcal{C}(G, p)$ contain all colourings of $G$ using at most $p$ colours. We use the notation $\chi_{D}(G, p)$ to denote the number of equivalence classes in $\mathcal{C}(G, p)$ that contain only distinguishing colourings of $G$. Note that if $p<\chi_{D}(G)$, then $\chi_{D}(G, p)=0$. In [3], Cheng looks at the distinguishing chromatic number of $\alpha H$, where $H$ is a connected graph.

Remark 2.3 [3, Fact 1(c)] Let $G=\alpha H$, where $H$ is a connected graph. Then

$$
\chi_{D}(G)=\min \left\{p: \chi_{D}(H, p) \geqslant \alpha\right\} .
$$

This result easily generalizes to the union of an arbitrary collection of pairwise disjoint graphs. Note that the union of an arbitrary collection of graphs can always be written in the form $\bigcup_{i=1}^{k} \alpha_{i} G_{i}$, where each $G_{i}$ is connected, and each pair $G_{i}$ and $G_{j}$ are nonisomorphic for every $i \neq j$. 
Remark 2.4 Let $G_{1}, G_{2}, \ldots, G_{k}$ be pairwise disjoint graphs where each $G_{i}$ is a connected graph and each pair $G_{i}$ and $G_{j}$ are nonisomorphic for every $1 \leqslant i<j \leqslant k$. If $G=$ $\bigcup_{i=1}^{k} \alpha_{i} G_{i}$, then

$$
\chi_{D}(G)=\min \left\{p: \chi_{D}\left(G_{i}, p\right) \geqslant \alpha_{i}, \text { for all } 1 \leqslant i \leqslant k\right\} .
$$

If $\chi_{D}(G)$ is close to $|V(G)|$, then one may expect $\chi_{D}(H)$ to be close to $|V(H)|$ for every induced subgraph $H$ of $G$. This statement is made precise in the next result.

Lemma 2.5 Let $G$ be a graph, $H$ be an induced subgraph of $G$ and $0 \leqslant t \leqslant|V(H)|$.

(i) If $\chi_{D}(G) \geqslant|V(G)|-t$, then $\chi_{D}(H) \geqslant|V(H)|-t$.

(ii) If $\chi_{D}(H) \leqslant|V(H)|-t$, then $\chi_{D}(G) \leqslant|V(G)|-t$.

Proof. Lemma 2.1 implies that

$$
\chi_{D}(G)-\chi_{D}(H) \leqslant \chi_{D}(G \backslash H) \leqslant|V(G)|-|V(H)|,
$$

from which $(i)$ and $(i i)$ follow.

Using Lemma 2.5, a list of forbidden induced subgraphs can be obtained for graphs $G$ of order $n$ that satisfy $\chi_{D}(G) \geqslant n-2$.

Notation 2.6 Figures of graphs with dotted lines in this paper represent classes of graphs obtained by independently replacing each dotted line with either an edge or nonedge. For example, the class of graphs depicted in Lemma $2.7\left(F_{2}\right)$ consists of five nonisomorphic graphs.

Lemma 2.7 Let $G$ be a graph of order $n$. If $\chi_{D}(G) \geqslant n-1$, then the following graphs cannot occur as induced subgraphs of $G$ :

$\left(F_{1}\right)$

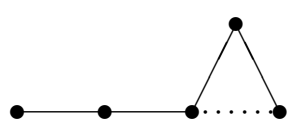

If $\chi_{D}(G) \geqslant n-2$, then the following graphs cannot occur as induced subgraphs of $G$ :

$\left(F_{2}\right)$

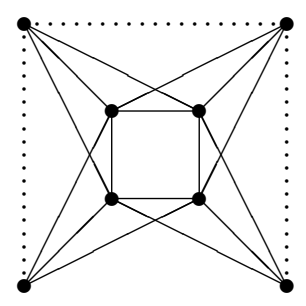

$\left(F_{3}\right)$

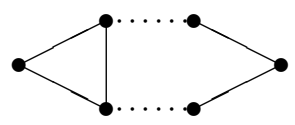

$\left(F_{4}\right)$

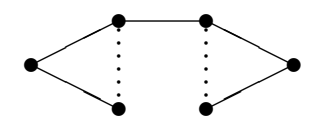


$\left(F_{5}\right)$

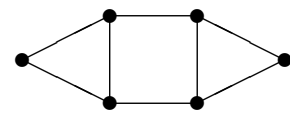

$\left(F_{8}\right)$

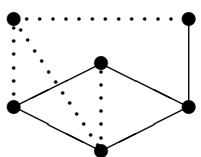

$\left(F_{11}\right)$

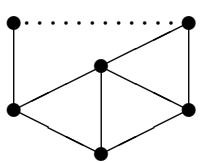

$\left(F_{14}\right)$

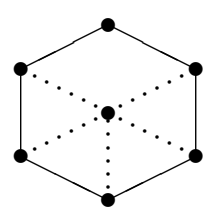

$\left(F_{17}\right)$

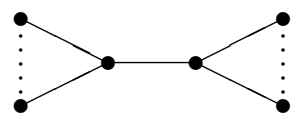

$\left(F_{6}\right)$

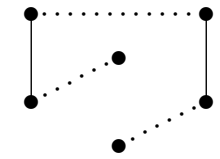

$\left(F_{9}\right)$

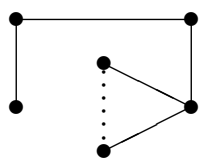

$\left(F_{12}\right)$

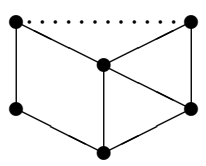

$\left(F_{15}\right)$

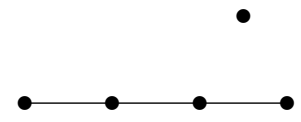

$\left(F_{16}\right)$

$\left(F_{13}\right)$

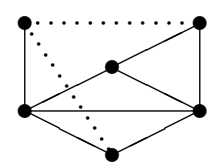

$\left(F_{10}\right)$

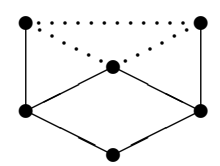

$\left(F_{7}\right)$

(F10)

$\left(F_{13}\right)$

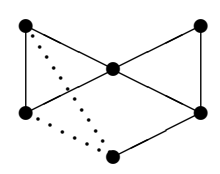

$\left(F_{18}\right)$

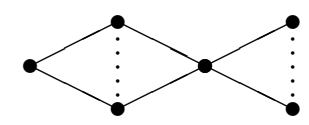

$\left(F_{19}\right)$

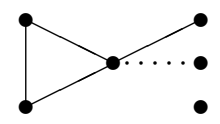

Proof. The Appendix provides distinguishing colourings (not necessarily optimal) for each of the graphs in classes $\left(F_{1}\right)-\left(F_{19}\right)$. The result now follows by Lemma 2.5. $\mathrm{Q}$

Given a distinguishing colouring $f$ of $G$, the next lemma provides a lower bound on the distinguishing chromatic number of a subgraph of $G$ induced by a collection of colour classes of $(G, f)$.

Lemma 2.8 Let $G$ be a graph and $f: V(G) \rightarrow\left\{1,2, \ldots, \chi_{D}(G)\right\}$ a distinguishing colouring of $G$. Let $\mathcal{C}_{i}$ be the set of vertices with colour $i$ and $A \subseteq\left\{1,2, \ldots, \chi_{D}(G)\right\}$. Then

$$
\chi_{D}\left(G\left[\bigcup_{i \in A} \mathcal{C}_{i}\right]\right) \geqslant|A| .
$$

In particular, if $S=\bigcup_{\left|\mathcal{C}_{i}\right|=1} \mathcal{C}_{i}$, then $G[S]$ is a complete multipartite graph. 
Proof. Let $H=G\left[\bigcup_{i \in A} \mathcal{C}_{i}\right]$ for some $A \subseteq\left\{1,2, \ldots, \chi_{D}(G)\right\}$. To derive a contradiction, suppose that $\chi_{D}(H)<|A|$. Let $g_{H}: V(H) \rightarrow A$ be a distinguishing colouring of $H$ using exactly $\chi_{D}(H)$ colours. We extend $g_{H}$ to a colouring of $G$ by defining

$$
g(x)=\left\{\begin{aligned}
g_{H}(x), & \text { if } x \in H \\
f(x), & \text { otherwise }
\end{aligned}\right.
$$

As $g$ colours $G$ with fewer than $\chi_{D}(G)$ colours, it cannot be distinguishing. Therefore, there is a nontrivial automorphism $\sigma: V(G) \rightarrow V(G)$ that preserves colours of $g$. Note that $x \in V(H)$ if and only if $\sigma(x) \in V(H)$. Let $\sigma_{H}$ be the restriction of $\sigma$ to $V(H)$. Then, $\sigma_{H}$ is a colour preserving automorphism for $\left(H, g_{H}\right)$. Since $g_{H}$ is a distinguishing colouring, it must be that $\sigma_{H}$ is the identity. Therefore, $\sigma$ pins every vertex in $H$. Now, viewing $\sigma$ as a colour preserving automorphism of $(G, f)$, it must be that $\sigma$ is the identity as $f$ is a distinguishing colouring of $G$. This is a contradiction.

Note that if $S=\bigcup_{\left|\mathcal{C}_{i}\right|=1} \mathcal{C}_{i}$, then $\chi_{D}(G[S]) \geqslant|S|$. Thus, by Theorem $1.1, G[S]$ is a complete multipartite graph.

The next lemma lists forbidden induced labelled subgraphs in $(G, f)$, when $f$ is a distinguishing colouring.

Lemma 2.9 Let $G$ be a graph and $f: V(G) \rightarrow\left\{1,2, \ldots, \chi_{D}(G)\right\}$ be a distinguishing colouring of $G$. Let $S$ be the set of all vertices belonging to singleton colour classes and suppose $\mathcal{C}_{r}$ is a doubleton colour class with $\mathcal{C}_{r}=\{x, y\}$. Then, for any $a, b, c \in S$, the following induced labelled subgraphs cannot occur in $(G, f)$ :
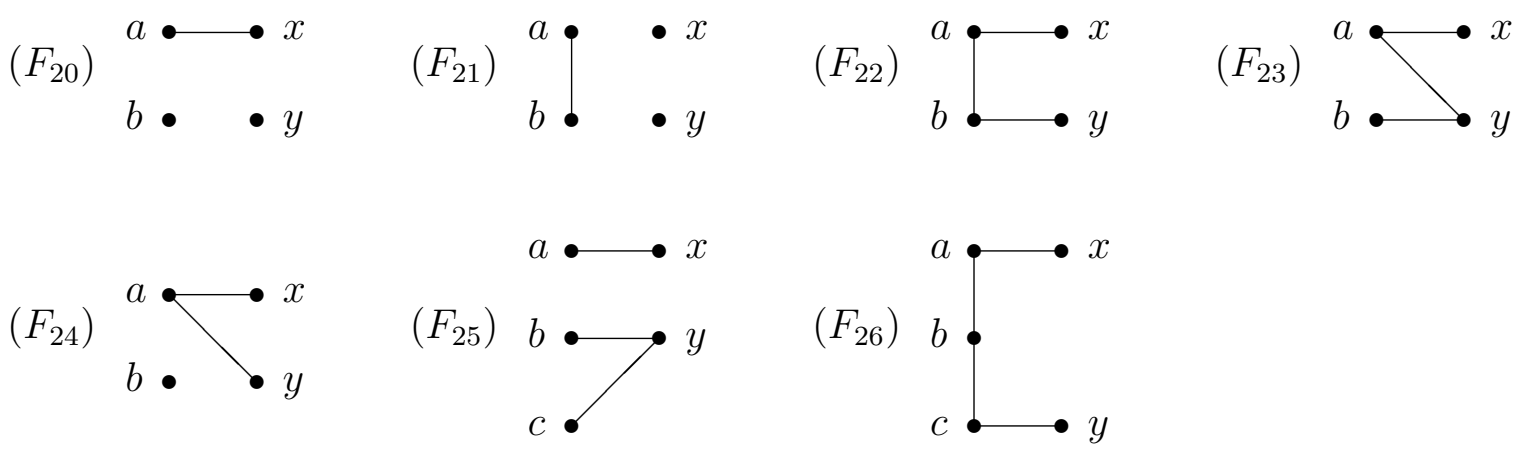

Proof. Let $H$ be one of $\left(F_{20}\right)-\left(F_{23}\right)$ and suppose $H$ occurs as an induced labelled subgraph in $(G, f)$. Then by Lemma 2.8 using $A=\{r, f(a), f(b)\}$, we have $\chi_{D}(H) \geqslant 3$, a contradiction as each of $\left(F_{20}\right)-\left(F_{23}\right)$ has distinguishing chromatic number two. A similar argument holds for $\left(F_{25}\right)$ and $\left(F_{26}\right)$, each of which has distinguishing chromatic number three.

Let $H$ be the induced subgraph $\left(F_{24}\right)$ and $\hat{f}: V(G) \rightarrow\left\{1,2, \ldots, \chi_{D}(G), \chi_{D}(G)+\right.$ $1\} \backslash\{f(a), f(b)\}$ the colouring:

$$
\hat{f}(u)=\left\{\begin{aligned}
\chi_{D}(G)+1, & \text { if } u=a \text { or } b \\
f(u), & \text { otherwise. }
\end{aligned}\right.
$$


Since $N_{H}(a) \neq N_{H}(b)$ and $N_{H}(x)=N_{H}(y), \hat{f}$ pins $a$ and $b$. But then any colour preserving automorphism of $(G, \hat{f})$ is also a colour preserving automorphism of $(G, f)$. Thus, $\hat{f}$ is a distinguishing colouring of $G$ using $\chi_{D}(G)-1$ colours, a contradiction.

In reference to Lemma 2.8, the next result provides information about the neighbourhoods of pairs of vertices that belong to the same part of the complete multipartite graph $G[S]$.

Lemma 2.10 Let $G$ be a graph and $f: V(G) \rightarrow\left\{1,2, \ldots, \chi_{D}(G)\right\}$ a distinguishing colouring of $G$. Let $S$ be the set of all vertices belonging to singleton colour classes and suppose $\mathcal{C}_{r}$ is a doubleton colour class with $\mathcal{C}_{r}=\{x, y\}$. Let $u, v \in S$ with $u \nsim v$. Then either

(a) $N_{G[S \cup\{x, y\}]}(u)=N_{G[S \cup\{x, y\}]}(v)$, or,

(b) $u, v$ belong to a part of $G[S]$ with size exactly two and $G[\{u, v, x, y\}] \cong 2 K_{2}$.

Proof. By Lemma $2.9\left(F_{23}, F_{24}\right)$, if $u \sim x$ and $u \sim y$, then $(a)$ is satisfied. By Lemma $2.9\left(F_{20}, F_{24}\right)$, if $u \nsim x$ and $u \nsim y$, then again $(a)$ is satisfied. By Lemma $2.9\left(F_{20}, F_{23}\right)$, if $u \sim x$ and $u \nsim y$, then we must have either $v \sim x$ and $v \nsim y$, or $G[\{u, v, x, y\}] \cong 2 K_{2}$. In the case that $v \sim x$ and $v \not y,(a)$ is satisfied. In the case that $G[\{u, v, x, y\}] \cong 2 K_{2}$ we have $v \nsim x$ and $v \sim y$. Furthermore, $u, v$ belong to a part of size exactly two in the graph $G[S]$, for otherwise there is a $c$ with $c \nsim u, c \nsim v$, and hence one of $\left(F_{20}\right),\left(F_{23}\right)$ or $\left(F_{25}\right)$ from Lemma 2.9 occurs.

\section{$3 \quad$ Graphs with $\chi_{D}(G)=|V(G)|-1$ or $\chi_{D}(G)=|V(G)|-2$}

In this section, we characterize the graphs that satisfy $\chi_{D}(G)=|V(G)|-1$ or $\chi_{D}(G)=$ $|V(G)|-2$ by using the tools developed in Section 2. First, we define an operation on graphs that allows us to "blow up" vertices of $G$ by replacing them by copies of arbitrary graphs. For any graph $G$ with vertices $\left(v_{1}, \ldots, v_{n}\right)$ and for any collection of vertex disjoint graphs $H_{1}, \ldots, H_{n}$, let $G\left(H_{1}, \ldots, H_{n}\right)$ denote the graph obtained from $G$ by replacing each $v_{i}$ with a copy of $H_{i}$ and replacing each edge $v_{i} v_{j}$ by $H_{i} \vee H_{j}$. Note that this operation is defined and used in [1]. If an $H_{i}$ is vacuous, i.e., $H_{i}=\emptyset$, then replacing $v_{i}$ by $\emptyset$ refers to deleting $v_{i}$ and all edges incident to it.

We next introduce some notation that allows us to partition the vertices belonging to singleton colour classes of a coloured graph $(G, f)$ according to their adjacency to nonsingleton colour classes.

Notation 3.1 Let $G$ be a graph, $f$ a distinguishing colouring of $G$ and $S$ the set of all vertices belonging to singleton colour classes of $(G, f)$. Let $\mathcal{A}=V(G) \backslash S$. For each $A \subseteq \mathcal{A}$, define $V_{A}=\{v \in S \mid v \sim A, v \nsim \mathcal{A} \backslash A\}$. Then $S$ can be partitioned as follows:

$$
S=\bigcup_{A \subseteq \mathcal{A}} V_{A}
$$


Furthermore, define $H_{A}=G\left[V_{A}\right]$ for each $A \subseteq \mathcal{A}$. If $A=\left\{a_{1}, \ldots, a_{k}\right\}$, we often write $V_{a_{1} \cdots a_{k}}$ to mean $V_{\left\{a_{1}, \ldots, a_{k}\right\}}$ and $H_{a_{1} \cdots a_{k}}$ to mean $H_{\left\{a_{1}, \ldots, a_{k}\right\}}$. In the case that $A=\emptyset$, we simply write $V_{0}$ and $H_{0}$.

Theorem 3.2 Let $G$ be a graph of order $n \geqslant 3$. Then $\chi_{D}(G)=n-1$ if and only if $G$ is the join of a complete multipartite graph (possibly vacuous) with one of the following:

(i) $2 K_{2}$, or,

(ii) $H \cup K_{1}$, where $H$ is a complete multipartite graph with at least two parts.

Proof. Observe that by Remarks 2.2 and 2.4, if $G$ is the join of a complete multipartite graph with one of $(i)$ or $(i i)$, then $\chi_{D}(G)=n-1$.

For the other direction, let $G$ be a graph of order $n \geqslant 3$ and let $f$ be a distinguishing colouring of $G$ using exactly $n-1$ colours. Let $S$ be the set of all vertices belonging to singleton colour classes and suppose $\mathcal{C}_{r}$ is a doubleton colour class with $\mathcal{C}_{r}=\{x, y\}$. Note that $|S|=n-2$ and $x \nsim y$. By Lemma $2.8, G[S]$ is a complete multipartite graph. By Lemma 2.10, for each part $P$ of $G[S]$, either

(a) $N_{G}(u)=N_{G}(v)$ for every $u, v \in P$, or

(b) $|P|=2$ and $G[P \cup\{x, y\}] \cong 2 K_{2}$.

By Lemma 2.7( $\left.F_{5}\right)$, there is at most one part satisfying $(b)$, say $Q$ where $Q$ could be vacuous. If $Q \neq \emptyset$, then by Lemma $2.7\left(F_{1}\right)$, we have $S \backslash Q \sim Q \cup\{x, y\}$. Therefore, $G \cong G[S \backslash Q] \vee 2 K_{2}$ and thus, $G$ has the form described in $(i)$.

Otherwise $Q=\emptyset$ and every part $P$ of $G[S]$ satisfies $(a)$. As described in Notation 3.1, define $V_{A}$ and $H_{A}$ for each $A \subseteq\{x, y\}$. Note that each of $H_{0}, H_{x}, H_{y}, H_{x y}$ is a complete multipartite graph and $G[S]=H_{0} \vee H_{x} \vee H_{y} \vee H_{x y}$. By Lemma $2.9\left(F_{22}\right)$, at least one of $V_{x}$ and $V_{y}$ is empty. Without loss of generality assume that $V_{y}=\emptyset$. By Lemma $2.9\left(F_{21}\right)$, $H_{0} \cong \bar{K}_{r}$, for some $r \geqslant 0$. Thus,

$$
G \cong H_{x y} \vee \hat{G}\left(\bar{K}_{r}, H_{x}, x, y\right),
$$

where $\hat{G}$ with vertices $\left(v_{0}, v_{1}, x, y\right)$ is the following labelled graph:

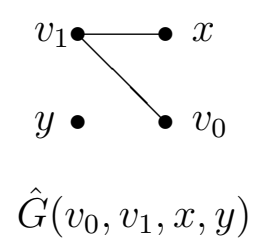

Note that the graph $H=G\left[V_{0} \cup V_{x} \cup\{x\}\right]$ is a complete multipartite graph. If $H$ has exactly one part, then $V_{x}=\emptyset$ implying that $G$ is a complete multipartite graph, contradicting that $\chi_{D}(G)=n-1$. Therefore $H$ has at least two parts and $G$ has the form described in $(i i)$. 
We now look at possible forms a graph $G$ of order $n$ may have under the condition $\chi_{D}(G)=n-2$. First, we define two labelled graphs $\hat{G}_{1}$ and $\hat{G}_{2}$ along with a class of labelled graphs $\mathcal{G}_{3}$ consisting of two nonisomorphic graphs.
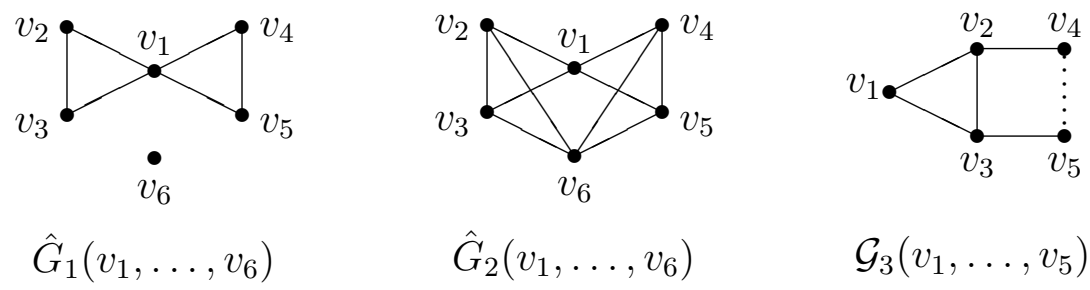

The labelled graphs $\hat{G}_{1}$ and $\hat{G}_{2}$ have vertices $\left(v_{1}, \ldots, v_{6}\right)$ while a labelled graph $\hat{G}_{3}$ belonging to the class $\mathcal{G}_{3}$ has vertices $\left(v_{1}, \ldots, v_{5}\right)$. The vertices of these graphs will be "blown up" to obtain graphs with distinguishing chromatic number $\chi_{D}(G)=|V(G)|-2$.

Theorem 3.3 Suppose $H_{1}, H_{2}$ and $H_{3}$ are complete multipartite graphs (possibly vacuous) and let $r, s \geqslant 1$ be integers. Let $G$ be a graph of order $n \geqslant 4$ with $\chi_{D}(G)=n-2$. Then $G$ is the join of a complete multipartite graph (possibly vacuous) and one of the following:

(i) $C_{6}$

(iii) $2 K_{2} \vee 2 K_{2}$

(v) $\hat{G}_{2}\left(H_{1}, v_{2}, v_{3}, v_{4}, v_{5}, v_{6}\right)$

(vii) $C_{5}$ (ii) $2 K_{3}$

(iv) $\hat{G}_{1}\left(H_{1}, v_{2}, v_{3}, v_{4}, v_{5}, v_{6}\right)$

(vi) $P_{5}$

(viii) $\hat{G}_{3}\left(H_{1}, H_{2}, H_{3}, \bar{K}_{r}, \bar{K}_{s}\right)$, for $\hat{G}_{3} \in \mathcal{G}_{3}$

Proof. Let $G$ be a graph of order $n \geqslant 4$ and let $f$ be a distinguishing colouring of $G$ using exactly $n-2$ colours. Let $S$ be the set of all vertices belonging to singleton colour classes.

Suppose there is a colour class of $(G, f)$ containing three vertices, say $x_{1}, x_{2}, x_{3}$. As $f$ is distinguishing, we have $N_{G}\left(x_{1}\right) \neq N_{G}\left(x_{2}\right)$, and hence, there is a $v \in S$ such that without loss of generality $v \sim x_{1}$ and $v \nsim x_{2}$ hold. Now let $\hat{f}$ be the colouring $f$ with $x_{2}$ recoloured by $f(v)$. Note that every colour preserving automorphism $\sigma$ of $(G, \hat{f})$ pins $x_{2}$ as $N_{G}\left(x_{2}\right) \neq N_{G}(v)$ and $\left\{x_{1}, x_{2}, x_{3}\right\}$ forms an independent set in $G$. Thus, $v$ is also pinned by $\sigma$. As $f$ is distinguishing, both $x_{1}$ and $x_{3}$ are pinned by $\sigma$. Therefore, every distinguishing colouring that has a colour class of size three can be transformed into a distinguishing colouring with two doubleton colour classes.

Suppose $\mathcal{C}_{r}$ and $\mathcal{C}_{s}$ are distinct doubleton colour classes with $\mathcal{C}_{r}=\{x, y\}$ and $\mathcal{C}_{s}=$ $\{w, z\}$. Note that $|S|=n-4, x \nsim y$ and $w \nsim z$. By Lemma 2.8, $G[S]$ is a complete multipartite graph (possibly vacuous). By Lemma 2.10, for each part $P$ of $G[S]$, either

(a) $N_{G}(u)=N_{G}(v)$ for every $u, v \in P$,

(b) $P=\{a, b\}, G[P \cup\{x, y\}] \cong 2 K_{2}$, and $N_{G[S \cup\{w, z\}]}(a)=N_{G[S \cup\{w, z\}]}(b)$,

(c) $P=\{c, d\}, G[P \cup\{w, z\}] \cong 2 K_{2}$, and $N_{G[S \cup\{x, y\}]}(c)=N_{G[S \cup\{x, y\}]}(d)$, or 
(d) $|P|=2, G[P \cup\{x, y\}] \cong 2 K_{2}$, and $G[P \cup\{w, z\}] \cong 2 K_{2}$.

By Lemma $2.7\left(F_{5}\right)$, there is at most one part satisfying $(b)$, say $Q$, at most one part satisfying $(c)$, say $R$, and at most one part satisfying $(d)$, say $T$, where $Q, R$ and $T$ could be vacuous. Furthermore, if $T \neq \emptyset$, then every other part of $G[S]$ must satisfy $(a)$.

For convenience, we let $J_{1}=G \backslash\{w, z\}$ and $J_{2}=G \backslash\{x, y\}$. By Lemma 2.8, we have $\chi_{D}\left(J_{1}\right) \geqslant n-3$ and $\chi_{D}\left(J_{2}\right) \geqslant n-3$. Hence, each of $J_{1}$ and $J_{2}$ is either complete multipartite or one of the graphs in Theorem 3.2.

Case (1): $T \neq \emptyset$. In this case, $Q=R=\emptyset$. As $2 K_{2}$ is an induced subgraph of both $J_{1}$ and $J_{2}$, it must be that $J_{1} \cong 2 K_{2} \vee G[S \backslash T]$ and $J_{2} \cong 2 K_{2} \vee G[S \backslash T]$, where $G[S \backslash T]$ is a complete multipartite graph of order $n-6$. Thus, $S \backslash T \sim T \cup\{x, y, w, z\}$. Consider $G[T \cup\{x, y, w, z\}]$. By Lemma $2.7\left(F_{3}, F_{4}, F_{5}, F_{6}\right)$, either $G \cong G[S \backslash T] \vee C_{6}$ or $G \cong G[S \backslash T] \vee 2 K_{3}$. Thus, $G$ has the form described in $(i)$ or $(i i)$.

For the remainder of the proof we assume that $T=\emptyset$.

Case (2): $Q \neq \emptyset$ and $R \neq \emptyset$. As $2 K_{2}$ is an induced subgraph of both $J_{1}$ and $J_{2}$, it must be that $J_{1} \cong 2 K_{2} \vee G[S \backslash Q]$ and $J_{2} \cong 2 K_{2} \vee G[S \backslash R]$, where $G[S \backslash Q]$ and $G[S \backslash R]$ are complete multipartite graphs of order $n-6$. Thus, $S \backslash(Q \cup R) \sim Q \cup R \cup\{x, y, w, z\}$. Furthermore, $Q \sim\{w, z\}, R \sim\{x, y\}$ and $Q \sim R$. Consider $G[Q \cup R \cup\{x, y, w, z\}]$. By Lemma $2.7\left(F_{2}\right),\{x, y\} \sim\{w, z\}$. Hence, $G \cong H \vee\left(2 K_{2} \vee 2 K_{2}\right)$, for some complete multipartite graph $H$, and thus $G$ has the form described in (iii).

Case (3): $Q \neq \emptyset$ and $R=\emptyset$. Suppose $Q=\{a, b\}$. Let $\hat{f}$ be a recolouring of $G$ using $n-3$ colours obtained by replacing the colour $f(a)$ with $f(b)$. Such a colouring cannot be distinguishing, and thus there is a colour preserving automorphism $\sigma$ of $(G, \hat{f})$ that maps $a$ to $b, x$ to $y$ and pins every vertex in $S \backslash Q$. Hence, $d_{G}(x)=d_{G}(y)$ and $d_{G}(a)=$ $d_{G}(b)$. Let $R_{1}=G[\{a, b, w, z\}]$ and $R_{2}=G[\{x, y, w, z\}]$. Note that $d_{R_{1}}(a)=d_{R_{1}}(b)$ and $d_{R_{2}}(x)=d_{R_{2}}(y)$.

As $2 K_{2}$ is an induced subgraph of $J_{1}$ it must be that $J_{1} \cong 2 K_{2} \vee G[S \backslash Q]$, where $G[S \backslash Q]$ is a complete multipartite graph of order $n-6$. Hence, $S \backslash Q \sim Q \cup\{x, y\}$ and each part $P$ in $G[S \backslash Q]$ satisfies $(a)$. Furthermore, $N_{R_{1}}(a)=N_{R_{1}}(b)$. By Lemma $2.9\left(F_{22}\right)$, there do not exist vertices $u, v$ in distinct parts of $S$ such that $G[\{u, v, w, z\}]$ is isomorphic to the graph in $\left(F_{22}\right)$. Thus, we may assume without loss of generality that for every part $P$ of $G[S]$ (including $Q$ ), if $P \sim z$ then $P \sim w$. As $R_{1} \neq 2 K_{2}, J_{2}$ is either a complete multipartite graph or $J_{2} \cong H_{2} \vee\left(H_{3} \cup\{z\}\right)$ for complete multipartite graphs $H_{2}$ and $H_{3}$. Let $\hat{V}$ consist of the parts $P$ in $G[S \backslash Q]$ that satisfy $P \sim z$ and let $H=G[\hat{V}], H^{c}=G[(S \backslash Q) \backslash \hat{V}]$. Given the structure of $J_{2}$, the graph $G\left[H^{c} \cup\{w\}\right]$ is complete multipartite.

By Lemma $2.7\left(F_{6}, F_{7}\right)$, we require $d_{R_{1}}(a) \neq 0$ and $d_{R_{2}}(x) \neq 0$. If $d_{R_{1}}(a)=2$ then by Lemma $2.7\left(F_{5}, F_{7}\right)$ we have $d_{R_{2}}(x)=2$. Therefore,

$$
G \cong H \vee \hat{G}_{2}\left(G\left[H^{c} \cup\{w\}\right], a, x, b, y, z\right),
$$

and thus $G$ has the form given in $(v)$.

Now, suppose $d_{R_{1}}(a)=1$. Then $Q \sim w$, otherwise $Q \sim z$ would imply $Q \sim w$ contradicting that $d_{R_{1}}(a)=1$. By Lemma $2.7\left(F_{13}\right)$, it must be the case that $d_{R_{2}}(x)=1$. By Lemma $2.7\left(F_{9}\right)$, we have either $w \sim\{x, y\}$ or $z \sim\{x, y\}$. In the case that $w \sim\{x, y\}$,

$$
G \cong H \vee \hat{G}_{1}\left(G\left[H^{c} \cup\{w\}\right], a, x, b, y, z\right),
$$


and thus $G$ has the form given in $(i v)$. Otherwise, $z \sim\{x, y\}$ and by Lemma $2.7\left(F_{14}\right)$ we have $H^{c}=\emptyset$, that is, $S \backslash Q \sim Q \cup\{x, y, w, z\}$. Therefore, $G \cong H \vee C_{6}$, and thus $G$ has the form described in $(i)$.

Case (4): $Q=\emptyset$ and $R \neq \emptyset$. This case is symmetric to Case (3).

For the remainder of the proof, we may assume that $Q=R=\emptyset$.

Case (5): In this case, every part $P$ of $G[S]$ satisfies $(a)$. As described in Notation 3.1, define $V_{A}$ and $H_{A}$ for each $A \subseteq\{x, y, w, z\}$. Note that each such $H_{A}$ is a complete multipartite graph, and furthermore, since each part of $G[S]$ satisfies $(a)$ we have

$$
G[S]=\bigvee_{A \subseteq\{x, y, w, z\}} G\left[H_{A}\right] .
$$

By Lemma $2.9\left(F_{22}\right)$, it must be the case that

$$
\left|V_{x} \cup V_{x w} \cup V_{x z} \cup V_{x w z}\right| \cdot\left|V_{y} \cup V_{y w} \cup V_{y z} \cup V_{y w z}\right|=0
$$

and

$$
\left|V_{w} \cup V_{x w} \cup V_{y w} \cup V_{x y w}\right| \cdot\left|V_{z} \cup V_{x z} \cup V_{y z} \cup V_{x y z}\right|=0 .
$$

Without loss of generality, we may assume

$$
V_{y}=V_{y w}=V_{y z}=V_{y w z}=V_{z}=V_{x z}=V_{x y z}=\emptyset .
$$

By Lemma $2.9\left(F_{21}\right)$, each of $H_{0}, H_{x}, H_{x y}, H_{w}, H_{w z}$ is an independent set, at most one of $V_{0}, V_{x}, V_{x y}$ can be nonempty, and at most one of $V_{0}, V_{w}, V_{w z}$ can be nonempty. Let $B=V_{0} \cup V_{x} \cup V_{x y}$ and $C=V_{0} \cup V_{w} \cup V_{w z}$. If $B=\emptyset$, define $W=\{w\}$ and $Z=\{z\}$; otherwise, fix $b \in B$ and let $\hat{f}_{1}$ be a recolouring of $G$ using $(n-3)$ colours by replacing the colour $f(b)$ with $f(w)=f(z)$. Since such a colouring cannot be distinguishing, there is a colour preserving automorphism of $\left(G, \hat{f}_{1}\right)$ that maps $b$ to either $w$ or $z$, and thus $N_{G}(b)$ is equal to either $N_{G}(w)$ or $N_{G}(z)$. Define independent sets

$$
W=\left\{\begin{array}{cl}
\{w\} \cup B, & \text { if } N_{G}(b)=N_{G}(w), \\
\{w\}, & \text { otherwise, }
\end{array} \quad \text { and } \quad Z=\left\{\begin{array}{cl}
\{z\} \cup B, & \text { if } N_{G}(b)=N_{G}(z), \\
\{z\}, & \text { otherwise. }
\end{array}\right.\right.
$$

Similarly, if $C=\emptyset$, define $X=\{x\}$ and $Y=\{y\}$; otherwise, fix $c \in C$ and let $\hat{f}_{2}$ be a recolouring of $G$ using $(n-3)$ colours by replacing the colour $f(c)$ with $f(x)=f(y)$. Since such a colouring cannot be distinguishing, there is a colour preserving automorphism of $\left(G, \hat{f}_{2}\right)$ that maps $c$ to either $x$ or $y$, and thus $N_{G}(c)$ is equal to either $N_{G}(x)$ or $N_{G}(y)$. Define independent sets

$$
X=\left\{\begin{array}{cl}
\{x\} \cup C, & \text { if } N_{G}(c)=N_{G}(x), \\
\{x\}, & \text { otherwise, }
\end{array} \quad \text { and } Y=\left\{\begin{array}{cl}
\{y\} \cup C, & \text { if } N_{G}(c)=N_{G}(y), \\
\{y\}, & \text { otherwise. }
\end{array}\right.\right.
$$

By construction, for each $u, v \in X, N_{G}(u)=N_{G}(v)$, and similarly for $Y, W$ and $Z$. Thus, we must have $X \sim W$ or $X \nsim W$, and similarly for pairs $\{X, Z\},\{W, Y\}$ and $\{Y, Z\}$. Then,

$$
G \cong H_{x y w z} \vee \hat{G}_{4}\left(H_{x w}, H_{x w z}, H_{x y w}, X, Y, W, Z\right),
$$

where $\hat{G}_{4}$ having vertices $\left(v_{1}, v_{2}, v_{3}, x, y, w, z\right)$ belongs to the class of labelled graphs $\mathcal{G}_{4}$ depicted as follows: 


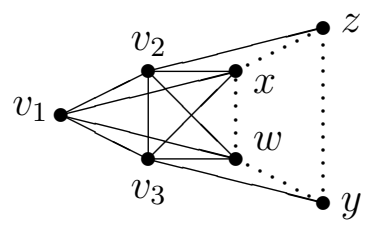

$\mathcal{G}_{4}\left(v_{1}, v_{2}, v_{3}, x, y, w, z\right)$

Case (5.1): If $X \sim W$, then $G \cong H_{x y w z} \vee \hat{G}_{3}\left(H_{1}, H_{2}, H_{3}, Z, Y\right)$ for $\hat{G}_{3} \in \mathcal{G}_{3}$, where

$$
\begin{aligned}
& H_{1}=\left\{\begin{aligned}
H_{x w} \cup X, & \text { if } X \nsim Z, W \sim Y, \\
H_{x w} \cup W, & \text { if } X \sim Z, W \nsim Y, \\
H_{x w} \cup X \cup W, & \text { if } X \nsim Z, W \nsim Y, \\
H_{x w}, & \text { if } X \sim Z, W \sim Y,
\end{aligned}\right. \\
& H_{2}=\left\{\begin{aligned}
H_{x w z} \cup X, & \text { if } X \sim Z, \\
H_{x w z}, & \text { if } X \nsim Z,
\end{aligned} \quad \text { and } \quad H_{3}=\left\{\begin{aligned}
H_{x y w} \cup W, & \text { if } W \sim Y, \\
H_{x y w}, & \text { if } W \nsim Y .
\end{aligned}\right.\right.
\end{aligned}
$$

Therefore, $G$ has the form described in (viii).

Case (5.2): Now, suppose that $X \not W$. If $X \nsim Z$ and $W \nsim Y$, then

$$
G \cong H_{x y w z} \vee \hat{G}_{3}\left(H_{x w} \cup X \cup W, H_{x w z}, H_{x y z}, Z, Y\right)
$$

for $\hat{G}_{3} \in \mathcal{G}_{3}$, and hence $G$ has the form described in (viii). Thus, we may assume that either $X \sim Z$ or $W \sim Y$ holds.

If $H_{x w} \neq \emptyset$, then by Lemma $2.7\left(F_{8}, F_{9}, F_{15}\right)$,

$$
G\left[H_{x w} \cup X \cup Y \cup W \cup Z\right] \cong P_{5} \quad \text { or } \quad G\left[H_{x w} \cup X \cup Y \cup W \cup Z\right] \cong C_{5} .
$$

By Lemma $2.7\left(F_{11}, F_{12}\right)$, we must have $H_{x w z}=H_{x y w}=\emptyset$ and hence $G$ has the form described in $(v i)$ or $(v i i)$.

Otherwise, $H_{x w}=\emptyset$. By Lemma $2.7\left(F_{10}\right)$ along with the assumption that either $X \sim Z$ or $W \sim Y$ holds, it must be the case that either $H_{x w z}=\emptyset$ or $H_{x y w}=\emptyset$. Without loss of generality, suppose $H_{x y w}=\emptyset$. Then

$$
G \cong\left\{\begin{aligned}
H_{x y w z} \vee \hat{G}_{3}\left(X \cup Z, \emptyset, H_{x w z}, Y, W\right), & \text { if } Y \nsim Z, \\
H_{x y w z} \vee \hat{G}_{3}\left(X, Z, H_{x w z}, Y, W\right), & \text { if } Y \sim Z, X \sim Z, \\
H_{x y w z} \vee \hat{G}_{3}\left(\emptyset, W \cup Z, H_{x w z}, Y, X\right), & \text { if } Y \sim Z, X \nsim Z, Y \sim W,
\end{aligned}\right.
$$

for $\hat{G}_{3} \in \mathcal{G}_{3}$, and hence $G$ has the form described in (viii).

We next determine conditions on graphs $G$ satisfying $\chi_{D}(G)=|V(G)|-2$ when $G$ is the join and union of complete multipartite graphs.

Lemma 3.4 Let $\Gamma_{0}, \Gamma_{1}$, and $\Gamma_{2}$ be complete multipartite graphs (possibly vacuous) and $G \cong \Gamma_{0} \vee\left(\Gamma_{1} \cup \Gamma_{2}\right)$. Then $\chi_{D}(G)=|V(G)|-2$ if and only if $\Gamma_{1} \cup \Gamma_{2}$ is isomorphic to:

(i) $\Gamma \cup K_{2}$, where $\Gamma$ is a complete multipartite graph with at least three vertices, 
(ii) $\Gamma \cup \bar{K}_{2}$, where $\Gamma$ is a complete multipartite graph with at least two parts, or

(iii) $2 K_{3}$.

Proof. If $G$ is one of the graphs in $(i)-($ iii $)$ then by Remarks 2.2 and 2.4 we have $\chi_{D}(G)=|V(G)|-2$.

For the other direction, let $G \cong \Gamma_{0} \vee\left(\Gamma_{1} \cup \Gamma_{2}\right)$ for complete multipartite graphs $\Gamma_{0}, \Gamma_{1}$, and $\Gamma_{2}$. By Remark 2.2, $\chi_{D}(G)=|V(G)|-2$ if and only if $\chi_{D}\left(\Gamma_{1} \cup \Gamma_{2}\right)=\left|V\left(\Gamma_{1} \cup \Gamma_{2}\right)\right|-2$. Without loss of generality we may assume that $\left|V\left(\Gamma_{1}\right)\right| \leqslant\left|V\left(\Gamma_{2}\right)\right|$. Note that $\left|V\left(\Gamma_{1}\right)\right| \geqslant 2$ for otherwise $G$ is a either a complete multipartite graph or a graph in Theorem 3.2. If $\left|V\left(\Gamma_{2}\right)\right| \geqslant 4$ and $\left|V\left(\Gamma_{1}\right)\right| \geqslant 3$, then $\chi_{D}\left(\Gamma_{1} \cup \Gamma_{2}\right) \leqslant\left|V\left(\Gamma_{1} \cup \Gamma_{2}\right)\right|-3$ by Remark 2.4. If $\left|V\left(\Gamma_{1}\right)\right|=\left|V\left(\Gamma_{2}\right)\right|=3$, then we require $\chi_{D}\left(\Gamma_{1} \cup \Gamma_{2}\right)=4$. If $\Gamma_{1} \approx \Gamma_{2}$, then $\chi_{D}\left(\Gamma_{1} \cup \Gamma_{2}\right)=3$. Furthermore,

$$
\chi_{D}\left(\bar{K}_{3} \cup \bar{K}_{3}\right)=6, \quad \chi_{D}\left(P_{3} \cup P_{3}\right)=3 \text { and } \chi_{D}\left(K_{3} \cup K_{3}\right)=4 .
$$

Therefore, $\Gamma_{1} \cup \Gamma_{2}$ satisfies (iii). Otherwise, we must have $\left|V\left(\Gamma_{1}\right)\right|=2$. If $\Gamma_{1} \cong K_{2}$, then either $\Gamma_{2} \cong \bar{K}_{2}$ or $\Gamma_{1}$ must have at least three vertices. Thus, $\Gamma_{1} \cup \Gamma_{2}$ satisfies either $(i)$ or $(i i)$. If $\Gamma_{1} \cong \bar{K}_{2}$, then $\Gamma_{2}$ must have at least two parts, and hence $G$ satisfies $(i i)$.

To characterize the graphs $G$ in Theorem 3.3 having distinguishing chromatic number $|V(G)|-2$ we define the following three labelled graphs.

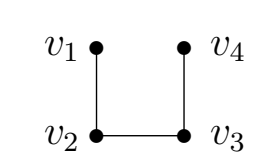

$\hat{G}_{5}\left(v_{1}, v_{2}, v_{3}, v_{4}\right)$

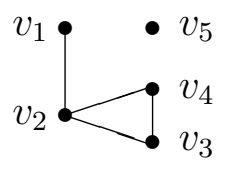

$\hat{G}_{6}\left(v_{1}, v_{2}, v_{3}, v_{4}, v_{5}\right)$

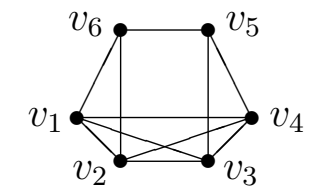

$\hat{G}_{7}\left(v_{1}, v_{2}, v_{3}, v_{4}, v_{5}, v_{6}\right)$

Furthermore, define $\hat{K}_{2}$ and $\hat{K}_{3}$ to be the labelled complete graphs of orders two and three respectively, where $\hat{K}_{2}\left(v_{1}, v_{2}\right)$ has vertices $\left(v_{1}, v_{2}\right)$ and $\hat{K}_{3}\left(v_{1}, v_{2}, v_{3}\right)$ has vertices $\left(v_{1}, v_{2}, v_{3}\right)$. In particular, if $H_{1}, H_{2}$ are nonvacuous complete multipartite graphs, then $\hat{K}_{2}\left(H_{1}, H_{2}\right)$ represents a complete multipartite graph with at least two parts.

Theorem 3.5 Let $G$ be a graph of order $n \geqslant 4$. Then $\chi_{D}(G)=n-2$ if and only if $G$ is the join of a complete multipartite graph (possibly vacuous) with one of the following:

(a) $P_{5}$

(c) $C_{6}$

(e) $\bar{K}_{r} \cup K_{2}$, for $r \geqslant 2$

(g) $\hat{K}_{3}\left(H_{1}, H_{2}, H_{3}\right) \cup K_{2}$ (b) $C_{5}$

(d) $2 K_{3}$

(f) $\hat{K}_{2}\left(\bar{K}_{r}, H_{1}\right) \cup K_{2}$, for $r \geqslant 2$

(h) $\hat{K}_{2}\left(H_{1}, H_{2}\right) \cup \bar{K}_{2}$ 
(i) $2 K_{2} \vee 2 K_{2}$

(k) $2 K_{2} \cup K_{1}$

(m) $\hat{G}_{3}\left(H_{1}, H_{2}, H_{3}, K_{1}, K_{1}\right)$, for $\hat{G}_{3} \in \mathcal{G}_{3}$

(o) $\hat{G}_{5}\left(K_{1}, H_{1}, H_{2}, K_{1}\right)$

(q) $\hat{G}_{7}\left(H_{1}, H_{2}, H_{3}, H_{4}, K_{1}, K_{1}\right)$ (j) $2 K_{2} \vee\left(\hat{K}_{2}\left(H_{1}, H_{2}\right) \cup K_{1}\right)$

(l) $\left(2 K_{2} \vee H_{1}\right) \cup K_{1}$

(n) $\hat{G}_{5}\left(H_{1}, H_{2}, K_{1}, K_{1}\right)$

(p) $\hat{G}_{6}\left(K_{1}, H_{1}, H_{2}, H_{3}, K_{1}\right)$

where each of $\mathrm{H}_{1}, \mathrm{H}_{2}, \mathrm{H}_{3}, \mathrm{H}_{4}$ is a nonvacuous complete multipartite graph.

Proof. For one direction, by Remark 2.2 and Theorem 1.1, we must show that if $G$ is one of $(a)-(q)$, then $G$ has distinguishing chromatic number $|V(G)|-2$. If $G$ is one of $(a)-(c)$, by Equation (1) the result holds. By Lemma 3.4, the result holds for $(d)-(h)$. By Remark 2.2, the result holds for $(i)$ and $(j)$ as these graphs are the join of two graphs in Theorem 3.2. One can also check using Remarks 2.2 and 2.4 that the result is true for $(k)$ and $(l)$.

Finally, let $G$ be one of $(m)-(q)$. Note that $G$ has an induced complete multipartite subgraph $H$ of order $|V(G)|-2$ such that each $u, v \in V(H)$ with $u \nsim v$ satisfies $N_{G}(u)=$ $N_{G}(v)$. Thus, each vertex in $V(H)$ requires a distinct colour giving $\chi_{D}(G) \geqslant|V(H)|=$ $|V(G)|-2$. Furthermore, $\chi_{D}\left(\hat{G}_{5}\right)=2, \chi_{D}\left(\hat{G}_{6}\right)=3, \chi_{D}\left(\hat{G}_{7}\right)=4$, and $\chi_{D}\left(\hat{G}_{3}\right)=3$ for each $\hat{G}_{3} \in \mathcal{G}_{3}$. As $G$ contains one of these graphs as an induced subgraph, by Lemma 2.5(ii), $G$ satisfies $\chi_{D}(G) \leqslant|V(G)|-2$, and hence $\chi_{D}(G)=|V(G)|-2$ whenever $G$ is of the form $(m)-(q)$.

For the other direction, let $G$ be a graph satisfying one of $(i)-($ viii $)$ in Theorem 3.3 such that $\chi_{D}(G)=|V(G)|-2$. We show $G$ has the form of one of $(a)-(q)$. To simplify the proof, we make use of the following claim that follows directly from Lemma 3.4.

Claim: Let $\chi_{D}(G)=|V(G)|-2$ and $G \cong \Gamma_{0} \vee\left(\Gamma_{1} \cup \Gamma_{2}\right)$, where $\Gamma_{0}, \Gamma_{1}, \Gamma_{2}$ are complete multipartite graphs (possibly vacuous). Then $G$ has the form described in one of $(d)-(h)$.

The graphs in Theorem 3.3 of the form $(i),(i i),($ iii $),(v i),(v i i)$ are listed in $(c),(d)$, $(i),(a),(b)$ respectively. The graphs in Theorem $3.3(i v)$ are listed in $(k)$ and $(l)$.

Let $G$ be a graph in Theorem 3.3(v) with $\chi_{D}(G)=|V(G)|-2$. Then $G \cong H_{0} \vee 2 K_{2} \vee$ $\left(H \cup K_{1}\right)$ for complete multipartite graphs $H_{0}$ and $H$. If $H$ has at most one part, then $\chi_{D}(G)=|V(G)|-1$, a contradiction. Therefore, $H$ has at least two parts and $G$ has the form listed in $(j)$.

Finally, let $G$ be a graph in Theorem 3.3(viii) with $\chi_{D}(G)=|V(G)|-2$. Then $G$ is the join of a complete multipartite graph $H_{0}$ (possibly vacuous) with $\hat{G}_{3}\left(H_{1}, H_{2}, H_{3}, \bar{K}_{r}, \bar{K}_{s}\right)$, where $\hat{G}_{3} \in \mathcal{G}_{3}, r, s \geqslant 1$ are integers and $H_{1}, H_{2}, H_{3}$ are complete multipartite graphs (possibly vacuous).

Case (1): $H_{1}=H_{2}=H_{3}=\emptyset$. In this case, $G$ is a complete multipartite graph and so $\chi_{D}(G)=|V(G)|$, a contradiction. 
Case (2): $H_{2}=H_{3}=\emptyset$ and $H_{1} \neq \emptyset$. In this case, $G$ satisfies the claim where $\Gamma_{0}=H_{0}, \Gamma_{1}=H_{1}$ and $\Gamma_{2}=G \backslash\left\{H_{0} \vee H_{1}\right\}$.

Case (3): $H_{1}=H_{3}=\emptyset$ and $H_{2} \neq \emptyset$. If $v_{4} \sim v_{5}$ in $\hat{G}_{3}$, then $G$ satisfies the claim with $\Gamma_{0}=H_{0} \vee \bar{K}_{r}, \Gamma_{1}=H_{2}$ and $\Gamma_{2}=\bar{K}_{s}$. If $v_{4} \nsim v_{5}$ in $\hat{G}_{3}$, the $G$ satisfies the claim with $\Gamma_{0}=H_{0}, \Gamma_{1}=H_{2} \vee \bar{K}_{r}$ and $\Gamma_{2}=\bar{K}_{s}$.

Case (4): $H_{1}=H_{2}=\emptyset$ and $H_{3} \neq \emptyset$. This case is symmetric to Case (3).

Case (5): $H_{1}=\emptyset$ and $H_{2}, H_{3} \neq \emptyset$.

Case (5.1): $\hat{G}_{3} \in \mathcal{G}_{3}$ has $v_{4} \sim v_{5}$. If $H_{2} \cong \bar{K}_{t}$ for some $t \geqslant 1$, then $G$ satisfies the claim with $\Gamma_{0}=H_{0} \vee\left(\bar{K}_{t} \cup \bar{K}_{s}\right), \Gamma_{1}=H_{3}$ and $\Gamma_{2}=\bar{K}_{r}$.

Otherwise, $H_{2}$ has at least two parts. By symmetry, we may also assume that $H_{3}$ has at least two parts. By Lemma $2.7\left(F_{19}\right)$ we must have $r=s=1$. Therefore, $G$ is of the form described in $(q)$.

Case (5.2): $\hat{G}_{3} \in \mathcal{G}_{3}$ has $v_{4} \not v_{5}$. By Lemma $2.7\left(F_{17}\right)$, either $r=1$ or $s=1$. Without loss of generality suppose that $r=1$. If $H_{2} \cong K_{1}$, then has the form described in $(n)$. Otherwise, $H_{2}$ has at least two vertices and by Lemma $2.7\left(F_{18}\right)$ we must have $s=1$, and hence $G$ has the form described in $(o)$.

Case (6): $H_{2}=\emptyset$ and $H_{1}, H_{3} \neq \emptyset$.

Case (6.1): $\hat{G}_{3} \in \mathcal{G}_{3}$ has $v_{4} \sim v_{5}$. By Lemma $2.7\left(F_{17}\right)$, either $H_{1} \cong K_{1}$ or $r=1$. If $H_{1} \cong K_{1}$ and $r \geqslant 2$, then by Lemma $2.7\left(F_{18}\right)$ we have $H_{3} \cong K_{1}$, and thus $G$ has the form described in $(n)$. Otherwise, $H_{1} \neq K_{1}$ and $r=1$. By Lemma 2.7 $\left(F_{18}\right)$ we have $s=1$, and hence $G$ has the form described in $(n)$.

Case (6.2): $\hat{G}_{3} \in \mathcal{G}_{3}$ has $v_{4} \not v_{5}$. If $H_{1} \cong \bar{K}_{t}$ for some $t \geqslant 1$, then $G$ satisfies the claim with $\Gamma_{0}=H_{0}, \Gamma_{1}=H_{3} \vee\left(\bar{K}_{t} \cup \bar{K}_{s}\right)$ and $\Gamma_{2}=\bar{K}_{r}$. Otherwise, $H_{1}$ has at least two parts. By Lemma 2.7( $\left.F_{16}\right), r=s=1$, and thus $G$ has the form described in $(p)$.

Case (7): $H_{3}=\emptyset$ and $H_{1}, H_{2} \neq \emptyset$. This case is symmetric to Case (6).

Case (8): $H_{1}, H_{2}, H_{3} \neq \emptyset$. In this case, $G$ has the form described in $(m)$.

\section{Acknowledgements}

We would like to thank the referees for constructive comments that improved the quality of this paper.

\section{References}

[1] N. Alon, J. Pach and J. Solymosi. Ramsey-type theorems with forbidden subgraphs. Combinatorica, 21(2):155-170, 2001.

[2] J.A. Bondy and U.S.R. Murty. Graph Theory. Springer, GTM 244, 2008.

[3] C.T. Cheng. On computing the distinguishing and distinguishing chromatic numbers of interval graphs and other results. Discrete Math., 309(16):5169-5182, 2009.

[4] J.O. Choi, S.G. Hartke and H. Kaul. The distinguishing chromatic number of Cartesian products of graphs. SIAM J. Discrete Math., 24(1):82-100, 2010. 
[5] K.L. Collins, M. Hovey and A.N. Trenk. Bounds on the distinguishing chromatic number. Electron. J. Combin., 16:\#R88, 2009.

[6] K.L. Collins and A.N. Trenk. The distinguishing chromatic number. Electron. J. Combin., 13:\#R16, 2006.

[7] E.M. Eschen, C.T. Hoàng, R. Sritharan and L. Stewart. On the complexity of deciding whether the distinguishing chromatic number of a graph is at most two. Discrete Math., 311(6):431-434, 2011.

[8] J. Jerebic, and S. Klavžar. The distinguishing chromatic number of Cartesian products of two complete graphs. Discrete Math., 310(12):1715-1720, 2010.

[9] W. Klöckl. On distinguishing and distinguishing chromatic numbers of hypercubes. Discuss. Math. Graph Theory, 28(3):419-429, 2008.

[10] C. Laflamme and K. Seyffarth. Distinguishing chromatic numbers of bipartite graphs. Electron. J. Combin., 16:\#R76, 2009.

[11] A.K. Lal and B. Bhattacharjya. Breaking the symmetries of the book graph and the generalized Petersen graph. SIAM J. Discrete Math., 23(3):1200-1216, 2009.

[12] S. Negami and S. Sakurai. Distinguishing chromatic numbers of planar graphs. Yokohama Math. J., 55(2):179-188, 2010.

[13] M.E. Watkins and X. Zhou. Distinguishability of locally finite trees. Electron. J. Combin., 14:\#R29, 2007.

[14] J. Weigand and M.S. Jacobson. Distinguishing and distinguishing chromatic numbers of generalized Petersen graphs. AKCE J. Graphs Combin., 5(2):199-211, 2008.

\section{Appendix}

Each colouring depicted is a distinguishing colouring for every graph in that class. In some cases, a class of graphs in Lemma 2.7 is split up into multiple subclasses (for example, the class of graphs $\left(F_{7}\right)$ is partitioned into two subclasses: $\left(F_{7}^{1}\right)$ and $\left.\left(F_{7}^{2}\right)\right)$.

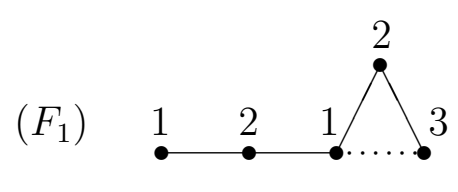

$\left(F_{2}\right)$
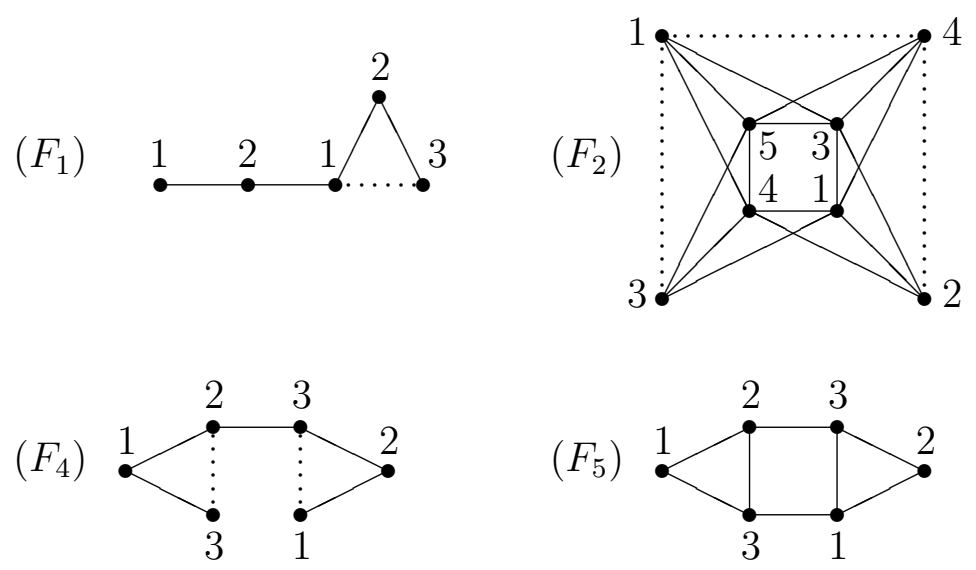

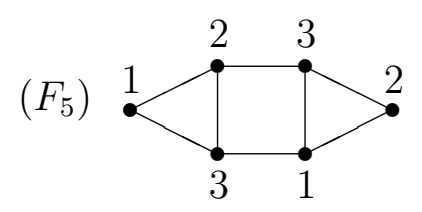

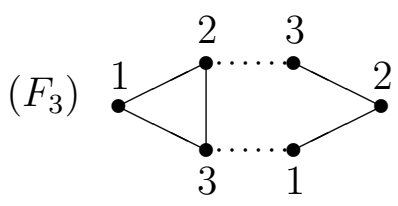

$\left(F_{6}\right)$

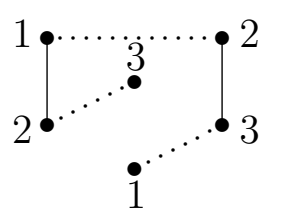



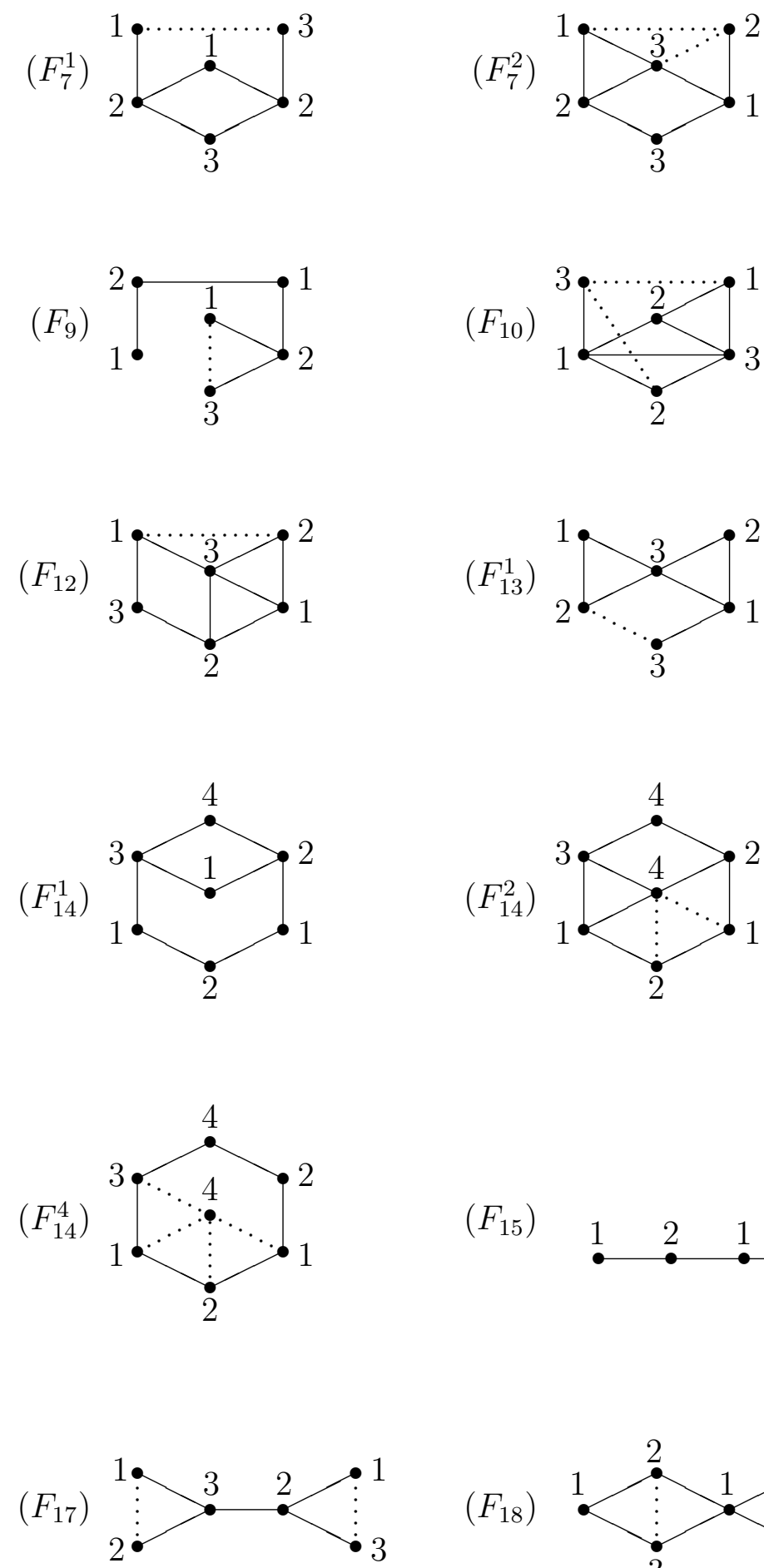
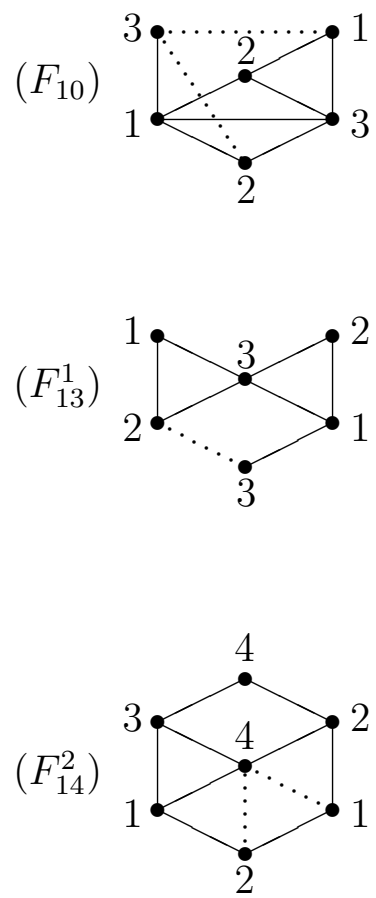

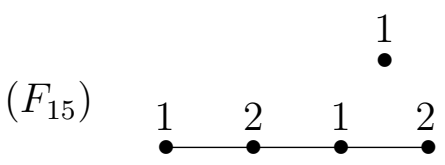

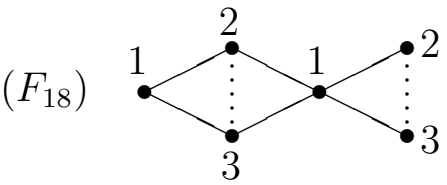

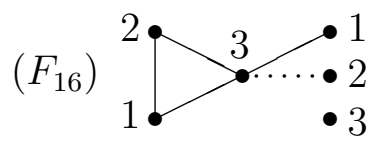
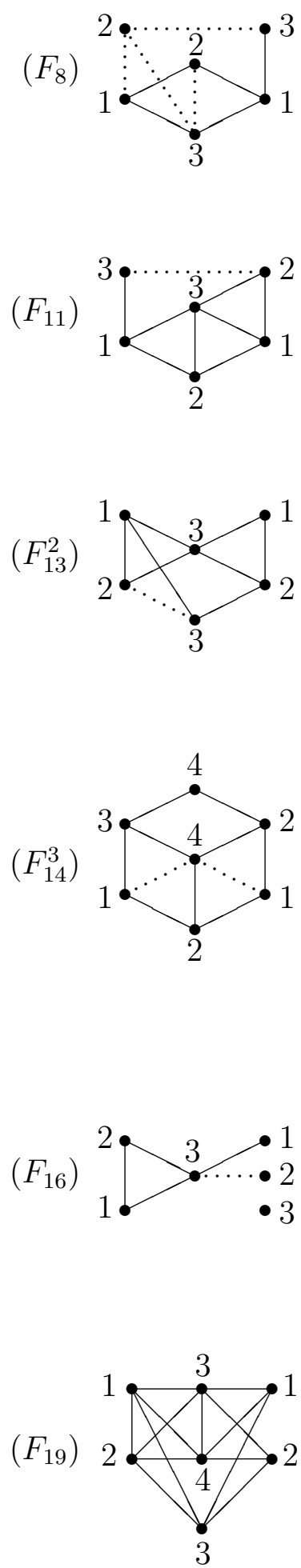